\title{
Hernia complicada en el canal de Nuck en el
} puerperio inmediato

\section{Complicated hernia in the canal of Nuck in immediate puerperium}

Adrián Palomino-Salas*, Gerardo E. Ornelas-Cortinas y Cinthia Y. Guillén-Gutiérrez

Departamento de Imagen Diagnóstica, Hospital Universitario Dr. José Eleuterio González, Universidad Autónoma de Nuevo León, Nuevo León,

Monterrey, México

\section{RESUMEN}

La hernia del canal de Nuck es un padecimiento infrecuente de la región inguinal de la mujer, que se presenta principalmente en infantes y raramente ocurre en adultas. El cuadro clínico consiste en la presencia de una masa en la región inguinal que se extiende hacia el labio mayor de la vagina. Es importante conocer la anatomía de la región inguinal de la mujer para poder hacer este diagnóstico. La valoración de estas pacientes debe hacerse de forma inmediata por el riesgo de estrangulamiento del saco herniario. El ultrasonido es el mejor método de imagen para su valoración en estas pacientes.

Palabras clave: Canal de Nuck. Hernia encarcelada. Hernia estrangulada. Ultrasonido

\section{ABSTRACT}

Canal of Nuck hernia is a rare condition of the inguinal region of the woman, more frecuently in infants, and rarely in adult women. The clinical presentation is a mass in the inguinal region that extends to the labia majora of the vagina. It is important to know the anatomy of the inguinal region of the women to make this diagnosis. The assessment of these patients should be done immediately due to the risk of strangulation of the hernia sac. Ultrasound is the best imaging method to assess these patients.

Keywords: Nuck canal. Incarcerated hernia. Strangulated hernia. Ultrasound 


\section{INTRODUCCIÓN}

El canal de Nuck es una extensión de peritoneo parietal en el canal inguinal femenino, que fue descrito en 1961 por el anatomista holandés Anton $\mathrm{Nuck}^{1-3}$. Esta estructura acompaña al ligamento redondo desde la base de las trompas de Falopio hasta el labio mayor; normalmente este debe obliterarse por completo durante el primer año de vida, pero cuando no ocurre así, su persistencia puede provocar una hernia o un quiste peritoneo-vaginal ${ }^{3}$.

La hernia del canal de Nuck es un padecimiento poco común ( $5 \%$ de las hernias en la región inguinal de la mujer). Cuando aparece suele ser difícil de diagnosticar y se confunde fácilmente con una hernia inguinal ${ }^{3}$. Debe evaluarse con urgencia debido a su posible encarcelamiento o estrangulación ${ }^{4}$. El método diagnóstico de elección es el ultrasonido².

\section{CASO CLÍNICO}

Mujer de 23 años, G4 P4, que acudió a la sala de urgencias del departamento de ginecología y obstetricia del Hospital Universitario Dr. José Eleuterio Gonzales, en el octavo día de puerperio fisiológico, presentando dolor en el hipogastrio y fiebre.

Se realizó ultrasonido pélvico por parte del servicio de radiología utilizando un equipo GE Logiq 7, con transductor lineal multifrecuencia, en el que se identificó el útero aumentado de tamaño, como puede verse en el puerperio, con imágenes ecogénicas en el endometrio, móviles con los cambios de posición de la paciente (Fig. 1). Se diagnosticó endometritis, por lo que fue internada para

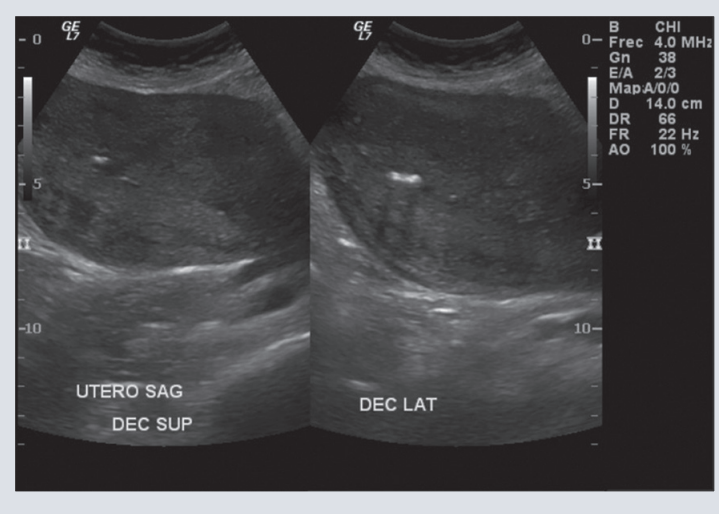

Figura 1. Útero en decúbito supino y decúbito lateral, que muestra imágenes ecogénicas móviles en el interior de la cavidad uterina, que corresponden a gas.

tratamiento y observación por el servicio de ginecología y obstetricia.

Tres días después de su ingreso presentó aumento de volumen, eritema y dolor punzante en la región inguinal izquierda que se extendía al labio mayor homolateral, y que empeoraba al realizar maniobras de Valsalva, sin atenuantes.

En la exploración física se identificó un aumento de volumen en la región inguinal izquierda, donde se palpó una masa de aproximadamente $2 \times 2 \mathrm{~cm}$, de consistencia firme, dolorosa a la palpación, con hiperemia de la zona y aumento de la temperatura de la piel. El abdomen se mostró blando y doloroso a la palpación del hipogastrio. La temperatura corporal era de $37.8^{\circ} \mathrm{C}$ y el resto de los signos vitales se encontraban normales.

Por parte del servicio de radiología se realizó ultrasonido de los tejidos blandos de la región inguinal izquierda utilizando un equipo Phillips EPIC 5G, con transductor lineal multifrecuencia, y se observó una hernia en el canal inguinal que contenía una imagen tubular correspondiente a la trompa de Falopio 


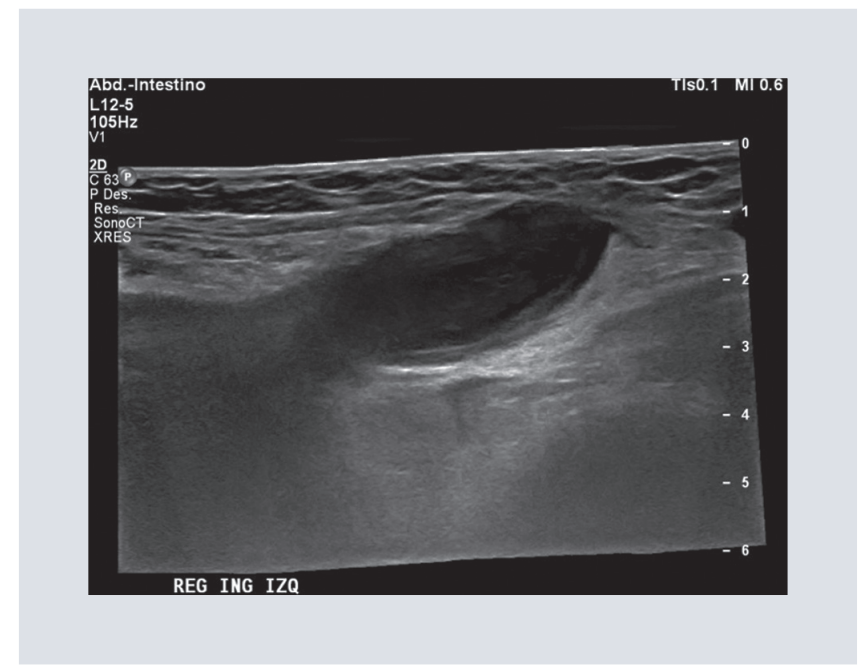

Figura 2. Región inguinal izquierda. Se observa la trompa de Falopio con engrosamiento de su pared, de ecogenicidad heterogénea e irregularidad de su pared interna, y borramiento de planos grasos adyacentes.

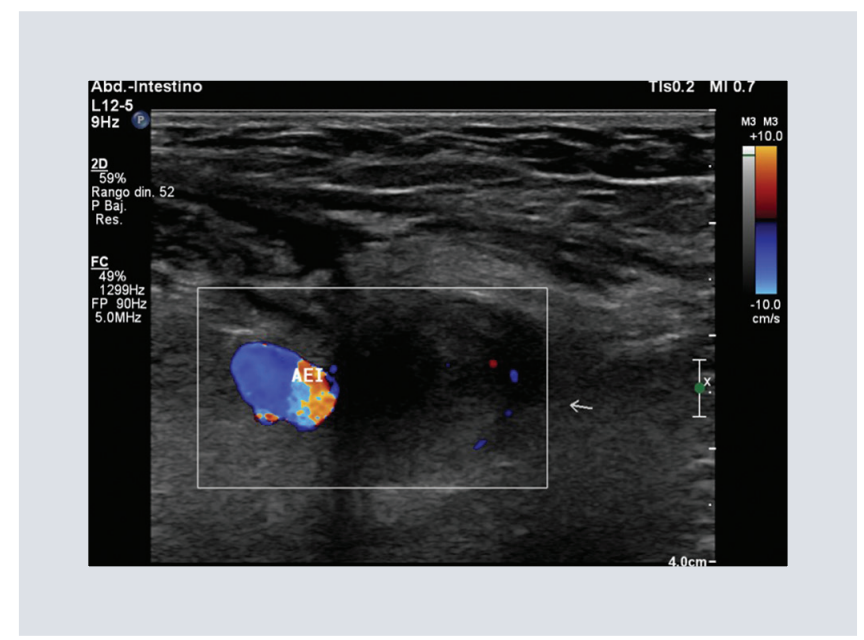

Figura 3. Ultrasonido Doppler que muestra la disposición del saco herniario lateral a los vasos epigástricos inferiores.

izquierda aumentada de tamaño, con engrosamiento de su pared y con contenido heterogéneo en su luz, de predominio anecoico, con reforzamiento acústico posterior, asociado a borramiento de los planos grasos adyacentes y a incremento del flujo en la exploración Doppler color (Fig. 2); dicha imagen se aproximaba al labio mayor homolateral a través del canal de Nuck (Fig. 3). Ambos ovarios estaban en su localización habitual y con características ecográficas normales (Fig. 4).

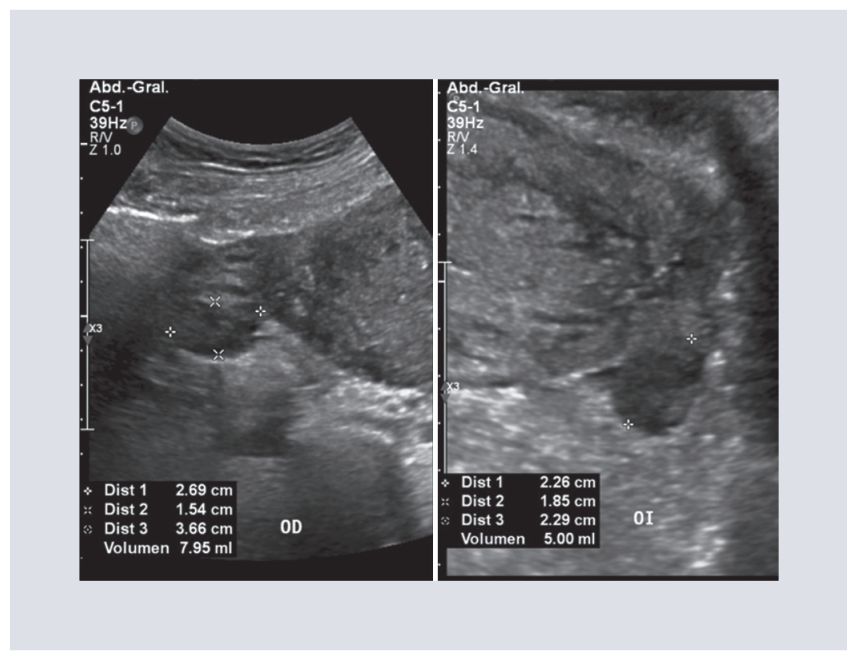

Figura 4. Ovarios de características normales.

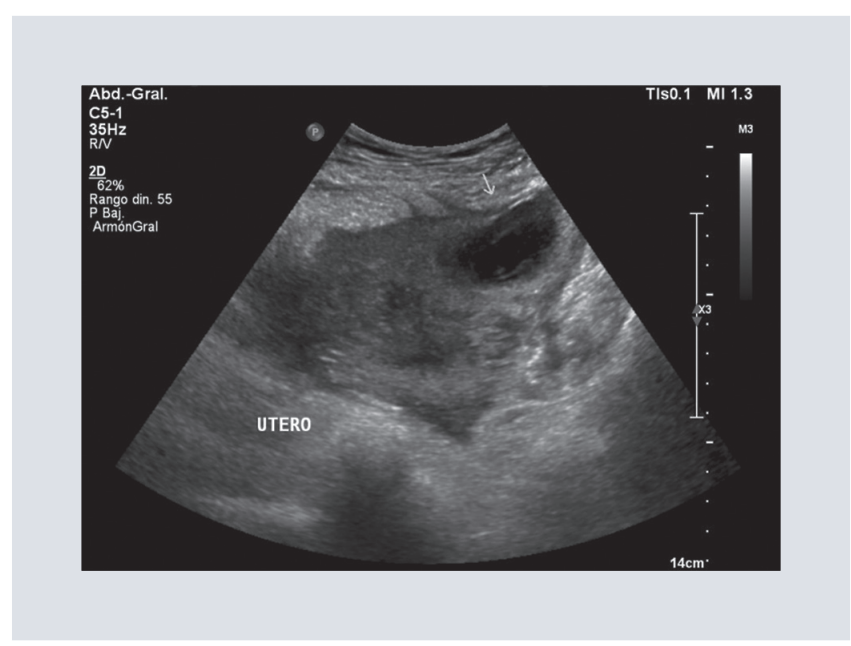

Figura 5. Istmo de la trompa de Falopio izquierda con datos de salpingitis.

Se diagnosticó hernia del canal de Nuck izquierdo conteniendo la trompa de Falopio homolateral, con datos de estrangulación condicionando salpingitis (Fig. 5). Posteriormente se realizó tomografía simple de abdomen para valoración preoperatoria y planeamiento quirúrgico, demostrando los mismos hallazgos (Fig. 6).

La paciente fue valorada por el servicio de ginecología y obstetricia para su tratamiento, y se realizaron histerectomía y reducción/ hernioplastia con salpingectomía izquierda. 

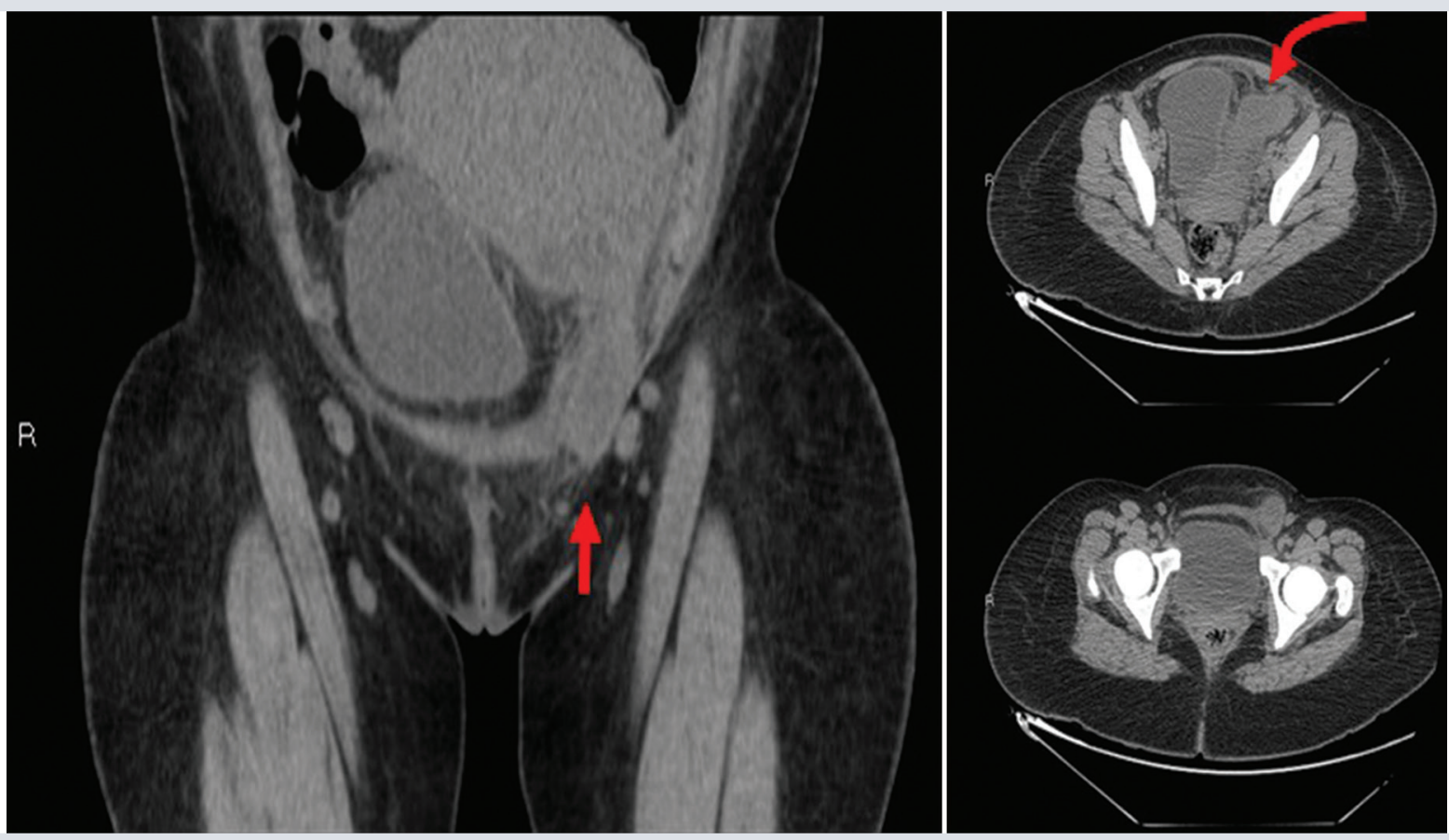

Figura 6. Tomografía computarizada simple de abdomen en reformateos coronal y axiales, que muestra una hernia del canal de Nuck del lado izquierdo, conteniendo la trompa de Falopio izquierda con datos de salpingitis.

El servicio de anatomía patológica y citopatología recibió las piezas quirúrgicas de útero, salpinge izquierda y ligamento redondo izquierdo, y su diagnóstico histopatológico fue útero (histerectomía) con datos de endometritis y deciduitis aguda, trompa de Falopio izquierda con datos de salpingitis aguda y crónica e hidrosalpinx, ligamento redondo con proceso inflamatorio agudo y crónico, edema y congestión vascular. La paciente evolucionó favorablemente y fue egresada sin complicaciones.

\section{DISCUSIÓN}

Las hernias de la región inguinal se dividen principalmente en directas e indirectas.
Las hernias inguinales directas, por definición, sobresalen a través del triángulo de Hesselbach, por encima del ligamento inguinal y medial al curso de los vasos epigástricos inferiores. Son más frecuentes en los varones, generalmente son adquiridas y aumentan en incidencia con la edad, como resultado del debilitamiento de la fascia transversal en el triángulo de Hesselbach ${ }^{5}$.

Las hernias inguinales indirectas surgen laterales y superiores al curso de los vasos epigástricos inferiores, laterales al triángulo de Hesselbach, y pasan a través del anillo inguinal interno para entrar en el conducto inguinal. En las mujeres, las hernias indirectas siguen el ligamento redondo hacia los labios mayores $^{6}$. El desarrollo anormal del canal de 
Nuck puede conducir a problemas que van desde el hidrocele asintomático hasta la hernia de órganos pélvicos ${ }^{2}$, como intestino, grasa omental, líquido, ovario, trompa de Falopio, rara vez útero y vejiga urinaria ${ }^{4}$. El intestino y la grasa omental son los más comúnmente implicados ${ }^{4}$. Del 15\% al 20\% de todas las hernias del canal de Nuck en la infancia contienen ovarios con o sin trompas de Falopio ${ }^{7,8}$.

Dentro de las complicaciones de las hernias se encuentran la encarcelación y la estrangulación. Una hernia se considera encarcelada o irreducible si el contenido no puede volver al abdomen de forma espontánea o por compresión. La hernia estrangulada se caracteriza por tener comprometido el suministro sanguíneo al contenido del saco herniario. Los signos de isquemia potencial incluyen líquido libre en el saco herniario, edema de la pared del órgano involucrado y ausencia de peristalsis intestinal ${ }^{6}$.

El ultrasonido tiene un papel esencial para la evaluación y el diagnóstico. Ofrece una valoración libre de radiación ionizante y con un costo menor que la realización de estudios de tomografía o resonancia magnética. Se debe valorar adecuadamente la región inguinal, caracterizando el contenido del saco herniario $^{2,7}$. La exploración de la región inguinal con ultrasonido ofrece una buena visualización de las estructuras inguinales en tiempo real, con posibilidad de realizar una exploración dinámica y así valorar la irreductibilidad de las hernias ${ }^{2,6}$.

Con el advenimiento de los escáneres de tomografía computarizada multidetector se visualiza mejor la anatomía de la región inguinal. Esto permite ver con mejor definición las estructuras pequeñas del canal inguinal y mejora el diagnóstico y la diferenciación entre los tipos de hernias ${ }^{5}$.

La resonancia magnética es una herramienta de imagen que ofrece un mayor campo de visión, pero tiene un costo más elevado y menor disponibilidad que el ultrasonido ${ }^{2}$.

Dentro del diagnóstico diferencial se deben considerar principalmente el quiste del canal de Nuck, las hernias inguinales directas, las adenomegalias inguinales y el absceso de un quiste de Bartolino.

\section{CONCLUSIONES}

El caso corresponde a una hernia del canal de Nuck estrangulada, que contenía la trompa de Falopio con salpingitis, lo cual es poco frecuente en pacientes adultas. Dicha patología es exclusiva de mujeres y su presentación más frecuente es una masa palpable en la región inguinal y el labio mayor en niñas menores de 5 años ${ }^{4,8-10}$.

La valoración por ultrasonido por un radiólogo capacitado es indispensable para realizar el diagnóstico oportuno de las hernias inguinales, así como identificar las estructuras involucradas en el saco herniario y detectar las complicaciones, para poder ofrecer el mejor tratamiento al paciente. La tomografía computarizada y la resonancia magnética ofrecen información importante para el diagnóstico, con mayor correlación anatómica, pero son estudios más costosos; además, la tomografía utiliza radiación ionizante y la resonancia magnética requiere mayor tiempo de 
adquisición de las imágenes, lo que puede limitar su uso.

\section{AGRADECIMIENTOS}

Al departamento de imagen diagnóstica del Hospital Universitario Dr. José Eleuterio González, por permitirnos utilizar sus equipos de ultrasonido y tomografía.

\section{FINANCIAMIENTO}

Los autores declaran que no hubo financiamiento.

\section{CONFLICTO DE INTERESES}

Los autores declaramos no tener conflictos de intereses.

\section{RESPONSABILIDADES ÉTICAS}

Protección de personas y animales. Los autores declaran que para esta investigación no se han realizado experimentos en seres humanos ni en animales.

Confidencialidad de los datos. Los autores declaran que han seguido los protocolos de su centro de trabajo sobre la publicación de datos de pacientes.

\section{Derecho a la privacidad y consentimiento} informado. Los autores han obtenido el consentimiento informado de los pacientes y/o sujetos referidos en el artículo. Este documento obra en poder del autor de correspondencia.

\section{BIBLIOGRAFÍA}

1. Oakes WJ. Indirect inguinal hernia of the urinary bladder through a persistent canal of Nuck: case report. Hernia. 2007;11:287-8.

2. Rees MA, Squires JE, Tadros S, Squires JH. Canal of Nuck hernia: a multimodality imaging review. Pediatric Radiol. 2017;47:893-8.

3. Martínez DG, Estela S, González N, Torreblanca CB, Sánchez AW. Caso clínico. Quiste del canal de Nuck asociado con hernia inguinal recurrente. Ginecol Obstet Mex. 2013;81:52-6.

4. Graul A, Ko E. Case report. Indirect inguinal hernia containing a Fallopian tube and ovary in a reproductive aged woman. Case Rep Obstet Gynecol. 2014;2014:10-3.

5. Joan HB, Yevgeniy A, Lawreance M, Francis JS. Diagnosis of inguinal region hernias with axial CT: the lateral crescent sign and other key findings. Radiographics. 2011;31:1-13.

6. Israel GM, Kirsch JD, Mathur M, Bokhari J, Scoutt LM. US of the inguinal canal: comprehensive review of pathologic processes with CT and MR imaging correlation. Radiographics. 2016;36:2028-48.

7. Thomas AK, Teague CT, Jancelewicz T. Canal of Nuck hernia containing pelvic structures presenting as a labial mass. Radiol Case Rep. 2018;13: 534-6.

8. Fakhry T, Albatanony A, Sabry A, Fawzy A. Incidence of sliding ovary and Fallopian tube in congenital inguinal hernia among female children. The Egyptian Journal of Surgery. 2017;36:1-5.

9. Karadeniz Cerit K, Ergelen R, Colak E, Dagli TE. Inguinal hernia containing uterus, fallopian tube and ovary in a premature newborn. Case Rep Pediatr. 2015;2015:807309.

10. Ming Y, Luo C, Chao H, Chu S. Inguinal hernia containing uterus and uterine adnexa in female infants: report of two cases. Pediatr Neonatol. 2011;52:103-5. 